\title{
Determinants of Patchy Metabolic Labeling in the Somatosensory Cortex of Cats: A Possible Role for Intrinsic Inhibitory Circuitry
}

\author{
S. L. Juliano, ${ }^{1,2}$ B. L. Whitsel, ${ }^{2}$ M. Tommerdahl, ${ }^{2}$ and S. S. Cheema ${ }^{2}$ \\ 'Department of Anatomy, USUHS, Bethesda, Maryland 20814, and 'Department of Physiology, University of North \\ Carolina, Chapel Hill, North Carolina 27514
}

\begin{abstract}
Despite repeated experimental demonstration that somatic stimulation leads to an intermittent, "column-like" pattern of 2-deoxyglucose (2DG) label in the somatosensory cortex, the functional significance of this pattern remains uncertain. A number of recent studies have suggested that the putative inhibitory neurotransmitter GABA may play an influential role in the cortical processing of sensory information. To test the possibility that GABA-mediated inhibitory processes might participate in the formation of the 2DG patches, the 2DG pattern obtained under "normal" experimental conditions was compared with the pattern observed when cortical inhibition was modified by topical application of the GABA antagonist, bicuculline methiodide (BIC). Under "normal" experimental conditions, we found that somatic stimulation led to an intermittent, patch like distribution of 2DG uptake in cat somatosensory cortex, which exhibited consistent features in animals studied using the same stimulus and experimental condition. Reconstructions of the stimulus-evoked activity patterns revealed that the label was confined to territories known to receive input from the stimulated body region and was organized into elongated strips. Topical application of BIC to the somatosensory cortex dramatically altered the dimension of the metabolic patches, which were often embedded in a field of elevated 2DG uptake. In BICtreated hemispheres the average width of 2DG patches was $1266 \mu \mathrm{m}$, whereas the average width of patches in the opposite untreated hemisphere (elicited by identical stimuli) was $713 \mu \mathrm{m}$. Unfolded maps of the labeling pattern revealed that in the BIC-treated hemispheres adjacent "strips" of 2DG label tended to fuse, leading to a less intermittent distribution than that observed in the untreated hemispheres. An important role for GABA in the formation of the normal cortical response to somatic stimulation is suggested.
\end{abstract}

Although it has been clearly established that somatic stimulation leads to patchy 2-deoxyglucose (2DG) labeling in the somatosensory cortex (Durham and Woolsey, 1978, 1985; Juliano et al., 1981; Hand, 1982; Juliano and Whitsel, 1985), questions remain as to the mechanism underlying $2 \mathrm{DG}$ patch formation.

\footnotetext{
Received July 16, 1987; revised June 10, 1988; accepted June 13, 1988.

We wish to thank Drs. Marc Peschanski and David Friedman for instructive comments on earlier versions of this manuscript. We also wish to thank Calvin Wong and Don Eslin for expert technical assistance. This research was supported by PHS NS-24014 (S.L.J.), DOD RO 7064 (S.L.J.), and PHS DE 07509 (B.L.W.)

Correspondence should be addressed to Dr. S. L. Juliano, Department of Anatomy, USUHS, 4301 Jones Bridge Road, Bethesda, MD, 20814.

Copyright (C) 1989 Society for Neuroscience $0270-6474 / 89 / 010001-12 \$ 02.00 / 0$
}

Although the idea that columns of cells in the neocortex act as functional units has guided research and thinking about cortical function for many years (for reviews, see Mountcastle, 1978; Jones, 1983), it has been difficult to demonstrate a relationship between the "patches" revealed with 2DG to the columns, which have been suggested to be functional units in the somatosensory cortex. As an example, experiments combining electrophysiology and metabolic mapping in the somatosensory cortex indicate that neurons "located between" as well as "in" 2 DG patches have similar response properties (Juliano et al., 1987).

The role of different neurotransmitters in the generation of cortical 2DG patches has not been previously explored. The possibility that GABA might play a role in the cortical processing of sensory stimuli is suggested by the recent demonstrations that iontophoretic application of a GABA antagonist can dramatically alter the response properties of specific categories of neurons in the visual and somatosensory cortex (Tsumoto et al., 1979; Dykes et al., 1984; Sillito, 1984; Alloway and Burton, 1986). In particular, the GABA antagonist bicuculline methiodide (BIC) allowed a population of neurons in both the visual and somatosensory cortex to respond to stimuli that were previously ineffective in driving them. These findings not only suggest that local inhibitory GABAergic circuits have the capacity to influence the sensory cortical representation of stimuli, but raise the possibility that GABA-mediated neurotransmission may modify the modular metabolic response detected by the 2DG mapping method of the somatosensory cortex to repetitive tactile stimulation.

Our selection of cats as experimental subjects was based on the fact that many of the published studies that evaluated the effects of BIC on individual cortical neurons used this species. Another reason for selecting the cat somatosensory cortex was that much of areas $3 \mathrm{~b}$ and $\mathrm{I}$ occupy an exposed position, thereby facilitating topical application of pharmacologic substances. Additionally, the metabolic modular organization of the cat somatosensory cortex has not yet been studied in detail. The aims of the current study were therefore (1) to determine the character of the metabolic labeling pattern evoked in the cat somatosensory cortex by repetitive somatic stimulation and (2) to determine the effects of the application of the GABA antagonist, BIC on the stimulus-evoked labeling pattern. A preliminary report of the results was presented previously (Juliano et al., 1984).

\section{Materials and Methods}

Eighteen male and female young adult cats weighing $1.5-2.5 \mathrm{~kg}$ were used in these studies; the animals were divided into 3 groups. The first group $(n=7)$ received $2 \mathrm{DG}$ injections alone, with no drug application, to establish the normal pattern of metabolic label produced in the so- 

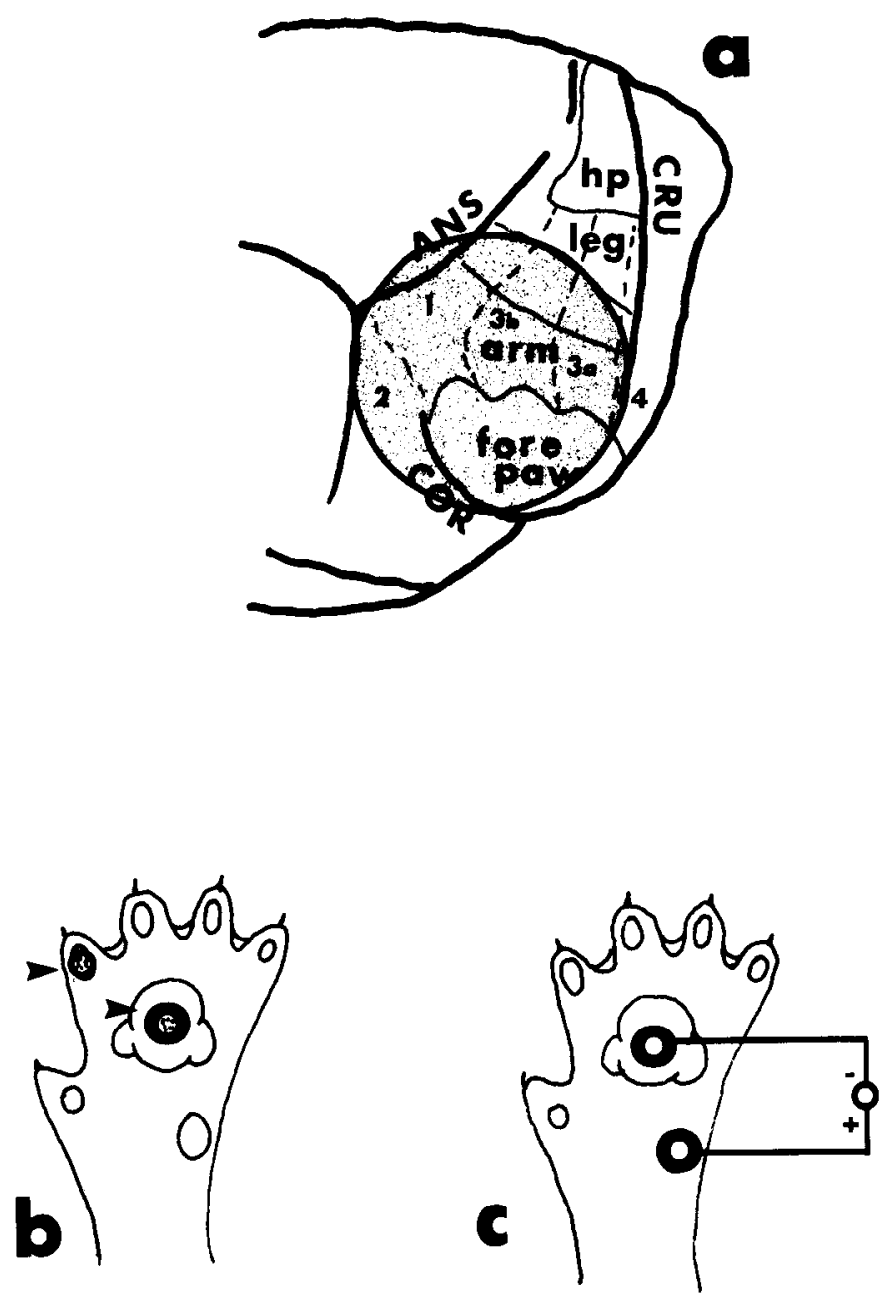

Figure 1. a, Schematic illustration of a lateral view of a cat brain (modified from McKenna et al., 1981). Shading indicates the approximate region of brain contacted by BIC in the drug studies. b) Illustration of regions of glabrous skin contacted by the flutter stimulator on digit 2 and the central pad. c) Placement of electrodes used for the electrocutaneous stimulation during the experiments using BIC. $C R U$, cruciate sulcus; $C O R$, coronal sulcus; $A N S$, ansate sulcus; $h p$, hindpaw.

matosensory cortex in response to somatic stimulation. In one animal of this group, a craniotomy was performed over the somatosensory cortex, but no drug was administered. This was done to ascertain the effect, if any, of the surgery on 2DG uptake. The second group of cats $(n=6)$ underwent the above surgical procedure and received topical applications of BIC prior to and during 2DG injection. Of this group, 2 animals did not receive somatic stimulation to the side of the body oppositc the drug application. This was done to evaluate the effect of $\mathrm{BIC}$ on 2DG incorporation in the somatosensory cortex, in the absence of stimulation. The third group of animals $(n=4)$ underwent the surgical procedure, were studied using extracellular electrophysiological recording methods, received topical applications of BIC, and then 2DG injections. The single- and multiple-unit recordings allowed determination of the effect of BIC on the responses of somatosensory neurons. In one additional animal, ${ }^{3} \mathrm{H}-\mathrm{BIC}$ was topically applied to the somatosensory cortex to reveal the extent and distribution of the absorption of BIC.

Surgery. The surgery was performed using halothane anesthesia and consisted of intubation with a tracheal cannula and the insertion of an i.v. catheter in the lateral saphenous vein. In the animals that underwent electrophysiological recordings and BIC application, an opening was made in the skull over primary somatosensory cortex (SI) and the dura removed (see Fig. 1). A Plexiglas chamber was secured over the opening with dental acrylic and the chamber filled with artificial CSF. A longlasting topical anesthetic ointment (dibucaine) was infused in all wound margins. At this point, neuromuscular blockade was achieved with gallamine triethiodide and the animal placed on artificial respiration; ex- pired $\mathrm{CO}_{2}$ and body temperature were monitored and maintained at normal levels. The halothane was withdrawn, and the animal received a mixture of nitrous oxide (70\%) and oxygen $(30 \%)$. The animal's head was held in place during the experiment with a device that fitted closely around the Plexiglas chamber but avoided direct contact with the subject. EEG was monitored during these experiments and provided an index of behavioral state: The periodic cycling between periods of synchronized and desynchronized activity exhibited by the subjects was typical of resting, unrestrained animals and was interpreted to indicate that the experimental conditions were not accompanied by pain or distress.

Application of BIC. BIC was applied topically to the somatosensory cortex of 11 cats. The BIC was dissolved in artificial CSF to achieve concentrations ranging from $10^{-5}$ to $10^{-7} \mathrm{M}$; these solutions were then placed in the recording chamber that was previously fixed to the skull. For one animal, ${ }^{3}$ II-BIC (New England Nuclear) was used. The ${ }^{3}$ II-BIC was reconstituted in artificial CSF to a concentration of $10^{-6} \mathrm{M}$ and applied to the brain in the manner described above.

Electrophysiology. Single- and multiple-unit recordings of neural activity were carried out as described previously (Juliano et al., 1987) Electrolytically sharpened, glass-insulated tungsten microelectrodes with tip diameters of $1-3 \mu \mathrm{m}$ were used. The recorded signals were amplified and visually displayed on an oscilloscope; the amplified signals were also fed into an audio amplifier. During the recording sessions, selected samples of the neural activity were saved on magnetic tape. Receptive fields (RFs) were initially determined using brushes and hand-held glass or wooden probes. In the experiments that assessed the effect of BIC on RF size, RFs were carefully mapped using von Frey hairs. The von Frey stimulus that elicited a consistent response was assessed prior to administration of BIC, and this same minimal von Frey stimulus was used to assess RF dimensions subsequent to BIC administration.

The 4 animals used for recording had a specially constructed chamber fitted with a Hamilton syringe, which allowed infusion of BIC into the CSF. This closed chamber was used to obtain highly stable extracellular recordings of neuronal discharge activity. This arrangement also permitted observation of the functional characteristics of single neurons and neuron clusters before and after BIC administration. In those experiments in which the animal subsequently underwent the 2DG procedure, the BIC was applied to the cortex at least 20 min prior to injecting the $2 \mathrm{DG}$, to allow for adequate diffusion into the cortex.

$2 D G$ experiments. The protocol followed for the $2 D G$ portion of these studies was similar to those published previously by this laboratory (e.g., Juliano et al., 1981) and that described initially by Sokoloff et al. (1977). A controllcd somatic stimulus was initiated approximately 10 min prior to the i.v. injection of the 2-deoxy-D-[1- $\left.{ }^{14} \mathrm{C}\right]$ glucose $(10 \mu \mathrm{Ci} /$ $100 \mathrm{gm}$ ). To obtain maximal information from a given experiment, some animals received bilateral stimuli, so both hemispheres would provide information. The stimuli were of 2 types: intermittent vertical displacement (flutter) and electrocutaneous. The flutter stimulator enabled the delivery of precisely controlled stimuli to small regions of skin. The stimulator carried a rounded Plexiglas probe $(1 \mathrm{~cm}$ in diameter) that was positioned just off the skin surface and oriented at a right angle. The stimulator was driven by a waveform generator and delivered controlled, intermittent sinusoidal displacements. The flutter stimuli were delivered at a frequency of $15 \mathrm{~Hz}$ with an amplitude of $0.5 \mathrm{~mm}$. Based on visual and manual inspection, the flutter stimulus did not set up detectable mechanical transients in the skin further than $1 \mathrm{~cm}$ from the cdge of the probe. Independent confirmation of this came from the recording experiments in which it was observed that neurons having RFs contiguous to, but nonoverlapping with, the stimulus site were not activated by the flutter probe. Figure 1 shows the locations of the skin sites stimulated. The electrocutaneous mode of stimulation was of interest for 2 reasons: (1) It does not set up mechanical transients, and (2) it is known to activate all large-diameter primary afferents nonselectively. Constant-current square-wave pulses $(0.9 \mathrm{~mA}$ in amplitude and $200 \mathrm{msec}$ in duration) were delivered repetitively (trains of 4 pulses/ sec; $100 \mathrm{msec}$ interpulse interval; $10 \mathrm{msec}$ intertrain interval). The stimuli were delivered using ECG electrodes attached to the surfaces to be stimulated. The electrocutaneous stimuli were always tested first on an investigator and subjectively judged to be nonnoxious; the stimuli evoked a sensation similar to that produced by a mechanical vibrator.

Each type of stimulus was applied repetitively for at least $10 \mathrm{~min}$ prior to $2 \mathrm{DG}$ administration and for $45 \mathrm{~min}$ after $2 \mathrm{DG}$ injection. The animal then received an overdose of pentobarbital $(35 \mathrm{mg} / \mathrm{kg})$ and was perfused transcardially with saline followed by $3.3 \%$ buffered formaldehyde. The brain was removed, blocked, and quickly frozen in Freon 

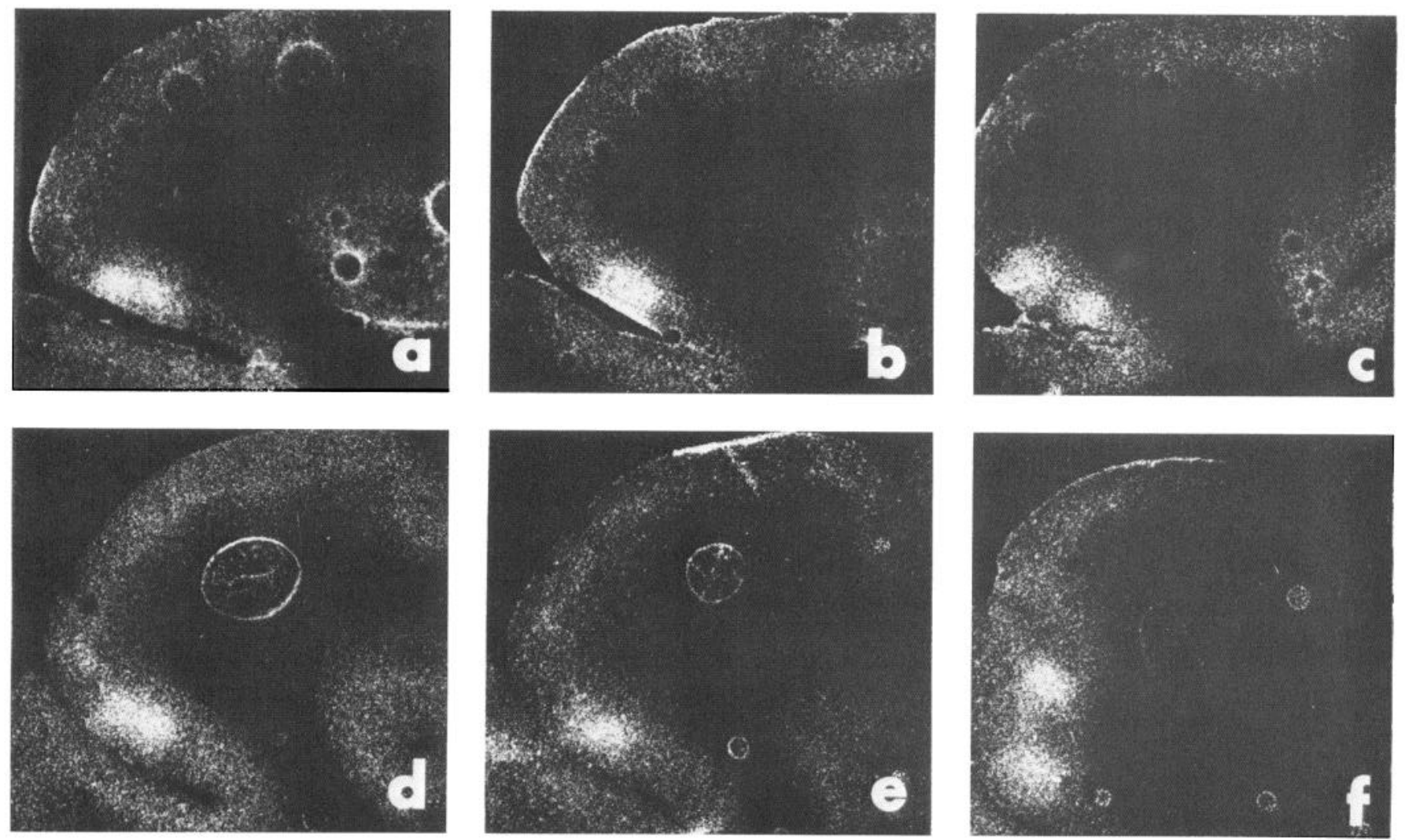

Figure 2. Series of autoradiographs taken from 2 different animals stimulated with a flutter stimulus on digit $2, a-c$ are from one animal, and $d$ $f$ are from a second. The autoradiographs represent a rostrocaudal distribution of activity taken at approximately the same locations in the 2 brains. A single patch of labeling can be seen in sections $a, d$ and $b, e$; the single patch subsequently forms 2 patches in sections $c$ and $f$. The sulcal patterns in the 2 brains are slightly different. The approximate rostrocaudal locations of these sections in a 2-dimensional reconstruction is shown in Figure 3 (arrows). These photographs are printed directly from the film autoradiographs, thus regions of high activity are white. Sections are taken in the coronal plane through the coronal sulcus.

22. Later, $20 \mu \mathrm{m}$ sections were cut in the coronal plane on a cryostat at $-16^{\circ} \mathrm{C}$. The tissue was then processed for autoradiography on SB5 film (Kodak). The sections from the brain that received ${ }^{3} \mathrm{H}$-BIC were exposed to LKB Ultrafilm for 1 week. After the autoradiographs were developed, the corresponding tissue sections were stained with thionin to allow identification of cortical laminae and cytoarchitecture.

Computer analysis. The autoradiographs were analyzed using a Micro PDP-11/23+-based computer-imaging system that digitizes standard video signals (Tommerdahl et al., 1985). For selected brains, a semiautomated system of generating 2-dimensional reconstructions from serial autoradiographs was used to view the spatial distribution of ${ }^{14} \mathrm{C}$-deoxyglucose concentration globally across the somatosensory cortex (Tommerdahl et al., 1985). The reconstruction process requires the user to enter with a bit-pad the inner (i.e., interface between gray and white matter) and outer (pial surface) boundaries of a selected region of cortex, along with specific points of reference (either cytoarchitectural or morphological) for a number of serial autoradiographs. The average ${ }^{14} \mathrm{C}$ value of the labeling in the tangential dimension is determined for each autoradiograph. The bin size for this value is variable, but measures approximately $50 \mu \mathrm{m}$. The resulting spatial histogram from each section is displayed as a vertical line of pixels on the video monitor; the bin height, which reflects ${ }^{14} \mathrm{C}$ concentration, is represented by pixel intensity. The lines are then stacked horizontally and aligned with respect to a selected point of reference. In this study the point of reference used to align the data was the fundus of the coronal sulcus.

\section{Results}

General characteristics of the stimulus-evoked 2DG labeling

\section{Tactile stimulation}

The metabolic labeling pattern evoked in SI by a cutaneous flutter stimulus was studied in 5 hemispheres. In all cases, the labeling occurred predominantly in the form of patches in cytoarchitectonic areas $3 \mathrm{~b}, 1$, and 2 . The ${ }^{14} \mathrm{C}$ concentration values of a given patch were found to be at least $60 \%$ above background, where background is considered to be the labeling value of the white matter. Cortical regions adjacent to or in between patches ranged between 20-30\% above background. Using our imaging system, patch width was measured by the following method. As indicated above, the activity values within a patch exceeded background, ranging between $60-90 \%$ above white matter values. Although patches were clearly visible by eye, pseudocolor enhancement of label exceeding background levels by $60 \%$ or greater enabled the definition of patch boundaries. Patches visualized in this manner were measured through lower layer III using a digitizing tablet. The average width of the patches differed depending on the site of the mechanical stimulation (i.e., digit 2 or central pad; see Fig. 6), and a range of widths existed for each stimulus condition. The average patch width for the digit 2-stimulated animals was $831 \mu \mathrm{m}$ (e.g., Figs. 2 and 6), with a range of $420-1120 \mu \mathrm{m}$. By contrast, the average patch width for central pad-stimulated animals was $594 \mu \mathrm{m}$ (e.g., Figs. 4 and 6 ), with a range of $420-848 \mu \mathrm{m}$. In general, the patches extended from lamina II to IV and only rarely lamina V. Occasional dense, band-like, tangential label was found in layer IV, predominantly in areas $3 b$ and 1 (see Fig. 4). In the 2 animals that received only a unilateral somatic stimulation, no patches were observed in the hemisphere contralateral to the unstimulated side.

The unfolded pattern of label was reconstructed using our computer-based reconstruction method (see Materials and 


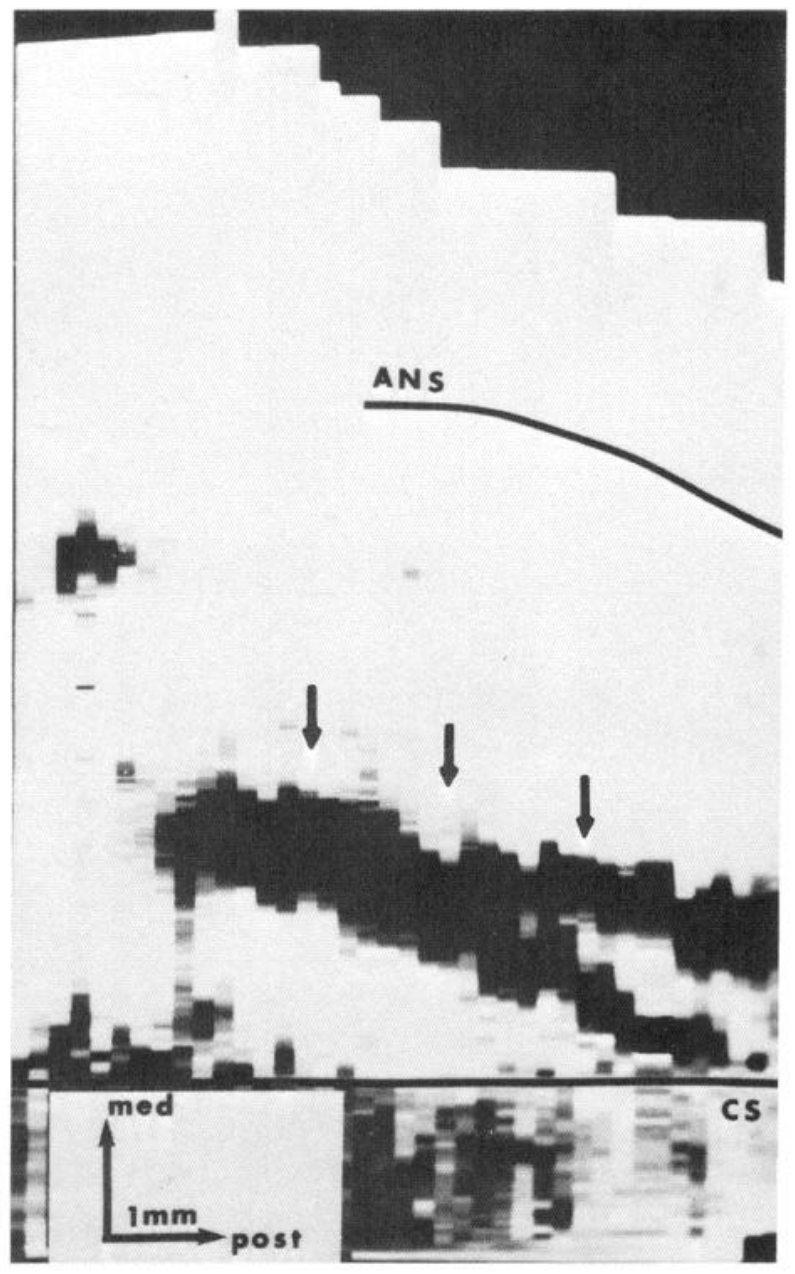

Figure 3. Digitized map of stimulus-evoked activity produced following flutter stimulation to digit 2 . The map was obtained by aligning digitized images on the coronal sulcus (CS); see Materials and Methods for details. The activity pattern consists of a rostrocaudally running strip that splits near its caudal extent. The arrows refer to the locations of the individual sections in Figure 2; the autoradiographs from the animal shown in Figure 2, $a-c$ produced this pattern of labeling.

Methods) for one of the flutter-stimulated animals. When viewed in this manner, the label clearly occurs as a strip or strips that run in a roughly rostrocaudal direction (Fig. 3).

Animals stimulated on different regions of the glabrous forepaw exhibited distributions of metabolic activity whose locations roughly correspond to the locations of the representations of those body parts in published maps of cat SI (McKenna et al., 1981; Felleman et al., 1983). For instance, the label evoked during stimulation of the ventral surface of digit 2 appears as a rostrocaudally running strip slightly medial to the fundus of the coronal sulcus. The major strip branches into 2 strips near the 3b-1 border.

The distribution of label shifted appreciably when the stimulus was applied to the central pad. As can be seen in Figure 4, the focus of labeling in those cases occupies a more medial location than that seen following stimulation of digit 2 (Fig. 2). Furthermore, when the same stimulus was applied to different animals, the distribution of label was found to be comparable from one animal to the next (see Fig. 2).

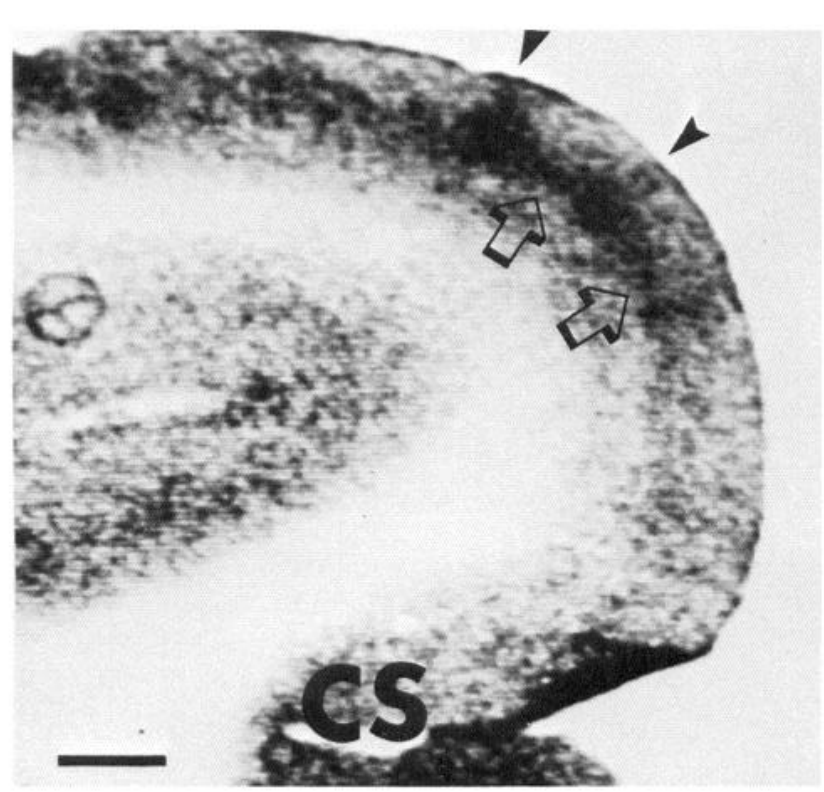

Figure 4. Distribution of 2DG labeling in a digitized section produced from flutter stimulation to the central pad. Arrowheads point to patches of activity. Open arrows indicate bandlike activity in layer IV. Section taken in a coronal plane through the coronal sulcus $(C S)$. Scale bar, 1 $\mathrm{mm}$.

\section{Electrocutaneous stimulation}

The distribution of label evoked in these experiments was similar in many respects to the distribution found after natural stimulation. The most obvious characteristic is that it occurs in patches extending from layer II to layer IV (see Fig. 5). Also evident in Figure 5 is the fact that the patches in adjacent sections line up to form strips. Differences between the patterns evoked by natural and electrocutaneous were also evident: the label produced from the electrocutaneous stimulation was more extensive than that found after the delivery of mechanical stirnuli and often appeared to contain components attributable to skin surfaces under and between both surface electrodes. This was anticipated because of the spread of current between the 2 surface electrodes. Figure 5, for example, demonstrates that patches occur buried in the coronal sulcus, which contains the representation of digit 2 , as well as more medially, on the gyral crown, where the central pad is represented.

Despite this difference in the extent of the labeling evoked by the 2 types of stimuli, the individual patches evoked by electrocutaneous stimulation are comparable in width to those found using the mechanical flutter stimulus. Like the patches evoked by mechanical skin stimulation, the electrocutaneous patches also exhibited a range of widths, but the patches found after digit 2 electrocutaneous stimulation tended to be wider than those evoked by electrocutaneous stimulation of the central pad. The width of the patches elicited by electrocutaneous stimulation to the central and pisiform pads were, on average, $627 \mu \mathrm{m}$ (range, 240-911 $\mu \mathrm{m}$ ), whereas for those produced from stimulation to digit 2 and the pisiform pad, the average width was $917 \mu \mathrm{m}$ (range, 420-1212 $\mu \mathrm{m}$ ) (see Fig. 6).

\section{Studies of BIC-treated hemispheres}

To test the possibility that local GABA-containing circuitry might participate in the formation of patchy metabolic patterns, 
we topically applied BIC to one hemisphere in 11 cats. 2DG label was produced by somatic stimuli delivered bilaterally, and the hemisphere that did not receive BIC was used for comparison with the drug-treated hemisphere.

\section{Controls}

In all animals, the EEG records allowed us to ascertain that subconvulsive doses of BIC were employed. We found that doses between $10^{-5}$ and $10^{-7} \mathrm{M}$ were effective in altering the cortical labeling pattern and did not cause seizures. Doses higher than $10^{-5} \mathrm{M}$ did produce seizure activity, and doses lower than $10^{-7} \mathrm{M}$ did not cause alterations in the metabolic labeling. In cases where seizures were elicited, they could be reversed by removing the BIC from the cortex and reapplying artificial CSF.

In 4 animals, cortical neuronal activity was monitored extracellularly with microelectrodes during the application of BIC. This analysis was carried out to ascertain the extent to which the presence of BIC in nonconvulsive doses (1) altered the spontaneous discharge activity of somatosensory neurons and (2) modified the capacity of peripheral stimulation to drive somatosensory cortical neurons. Multiunit activity was sampled for 10 cortical sites before, during, and after the application of BIC. The neural activity was monitored for about $65 \mathrm{~min}$, the approximate time period that BIC would be present during a 2DG experiment. A sample of the activity obtained from these neurons is shown in Figure 7. It was obvious that the application of a concentration of $10^{-6} \mathrm{M} \mathrm{BIC}$ to the cortex did not substantially alter the baseline level of spontaneous neuronal activity (compare before and after traces in Fig. 7) or the ability of neurons to respond reliably to somatic stimulation. Substantial changes were found, however, in the static response properties of the majority of the cells studied. For example, RF size increased considerably for 7 out of the 10 cortical sites analyzed in this manner. Figure 7 illustrates the increase in RF size observed at 2 locations studied $30 \mathrm{~min}$ after application of BIC to the cortex. The RF did not continue to enlarge after $30 \mathrm{~min}$ of exposure to BIC.

In one animal, a chamber was placed over SI and filled with artificial CSF and a 2DG experiment with somatic stimulation was carried out, but no BIC was applied to the cortex. This "control" experiment was conducted to determine if the character of the labeling would be altered by opening the skull and dura and attaching a chamber, in the absence of pharmacologic treatment. The character of the labeling was not found to differ from that of the animals with no chamber placement, suggesting that the procedure of opening the skull and dura did not alter the distribution of metabolic activity. This was also found to be true in our prior combination 2DG and recording experiments in primates (Juliano et al., 1987).

Previous use of the topical application of BIC (Rose and Blakemore, 1974) suggested that BIC gains access to all layers of the exposed cortex, since this study detected alterations in the response properties of single cells located at depths up to $1.6 \mathrm{~mm}$ from the pial surface. Despite this evidence, we felt it pertinent to ascertain that the concentrations of BIC used in our experimental conditions attained access to all cortical layers. To this end, the cortex of one animal was exposed to CSF containing $10^{-6} \mathrm{M}{ }^{3} \mathrm{H}-\mathrm{BIC}$. The tritiated BIC was applied in the same manner and for the same duration used for the animals that underwent a $2 D G$ experiment. Figure 8 clearly indicates that the ${ }^{3} \mathrm{H}-$ BIC penetrated the full thickness of the cerebral cortex and that it did not spread appreciably either into the underlying white matter or the cortical regions surrounding the edge of the chamber.

In 2 additional animals, BIC was applied to the somatosensory cortex but no somatic stimuli were delivered immediately prior to or for $45 \mathrm{~min}$ after the $2 \mathrm{DG}$ injection. These experiments were carried out to determine if the procedure of applying BIC to the somatosensory cortex by itself (in the absence of somatic stimuli) would directly elicit abnormal cortical metabolic activity patterns. Two different concentrations of BIC were used: $10^{-5}$ and $10^{-6} \mathrm{M}$. In both of these experiments (i.e., topical application of BIC during the administration of 2DG but without somatic stimulation), no unusual or generalized increases in metabolic activity in SI were found (see Fig. 9).

\section{Effects of BIC on $2 D G$ labeling}

Application of BIC to the somatosensory cortex caused changes in the metabolic pattern that varied with the dose. All effective doses led to abnormally wide patches in response to somatic
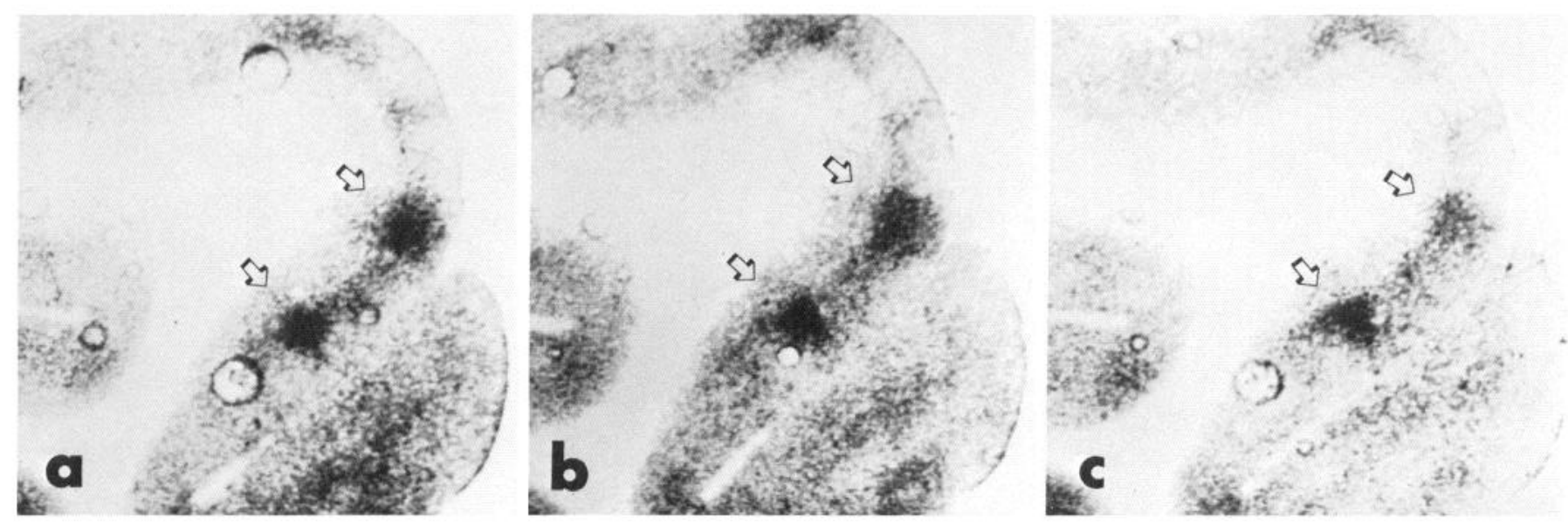

Figure 5. Distribution of 2DG labeling in 3 digitized sections from the same animal progressing rostrocaudally $(a-c)$. The animal was stimulated with electrocutaneous stimuli to digit 2 and the pisiform pad. The patches of $2 \mathrm{DG}$ activity can be followed through the sections, indicating the resultant pattern of labeling would form strips. Arrows indicate 2 prominent patches of labeling that can be followed from section to section. 

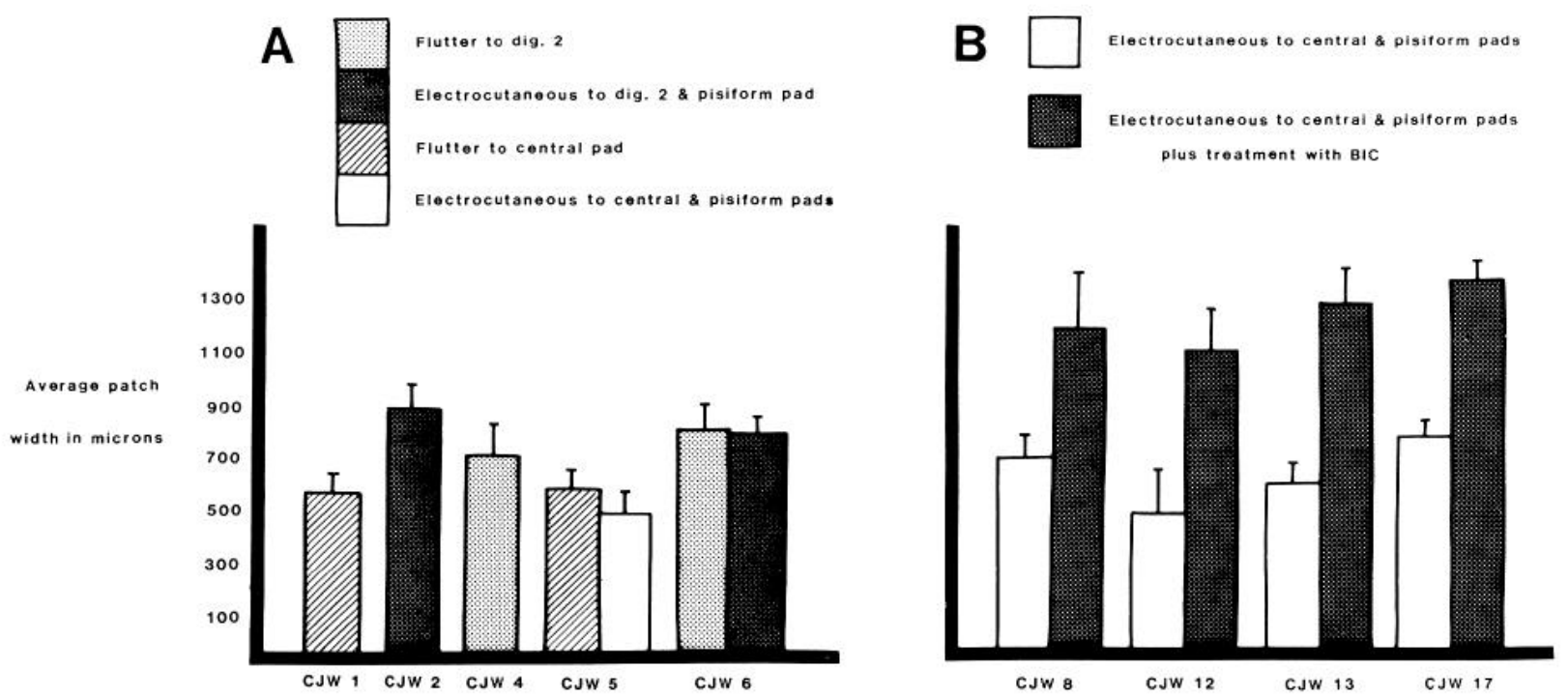

Figure 6. Average patch widths found in SI after different stimulus conditions. The animals represented in $A$ received somatic stimulation to the opposite forepaw. CJW 5 and 6 received different stimuli to both forepaws. The animals in $B$ were stimulated bilaterally with identical stimuli, but the right hemisphere was treated with BIC. CJW 8 and 17 received a $10^{-5} \mathrm{M}$ dose; CJW 12 and 13 received a $10^{-6} \mathrm{M}$ dose. The patch widths in the BIC-treated hemisphere were significantly wider $(p<0.05)$ than those in the opposite untreated hemispheres. SDs are indicated with bars.

stimulation. When higher doses $\left(10^{-5} \mathrm{M}\right)$ were used, the abnormally wide patches were found to extend through the full thickness of the cortex and to be embedded in a homogeneous field of increased label (e.g., Fig. 10; for this animal the cathode was placed on the pisiform pad). The homogeneous field of increased activity, although above background in optical density values,

\section{PRE BIC}

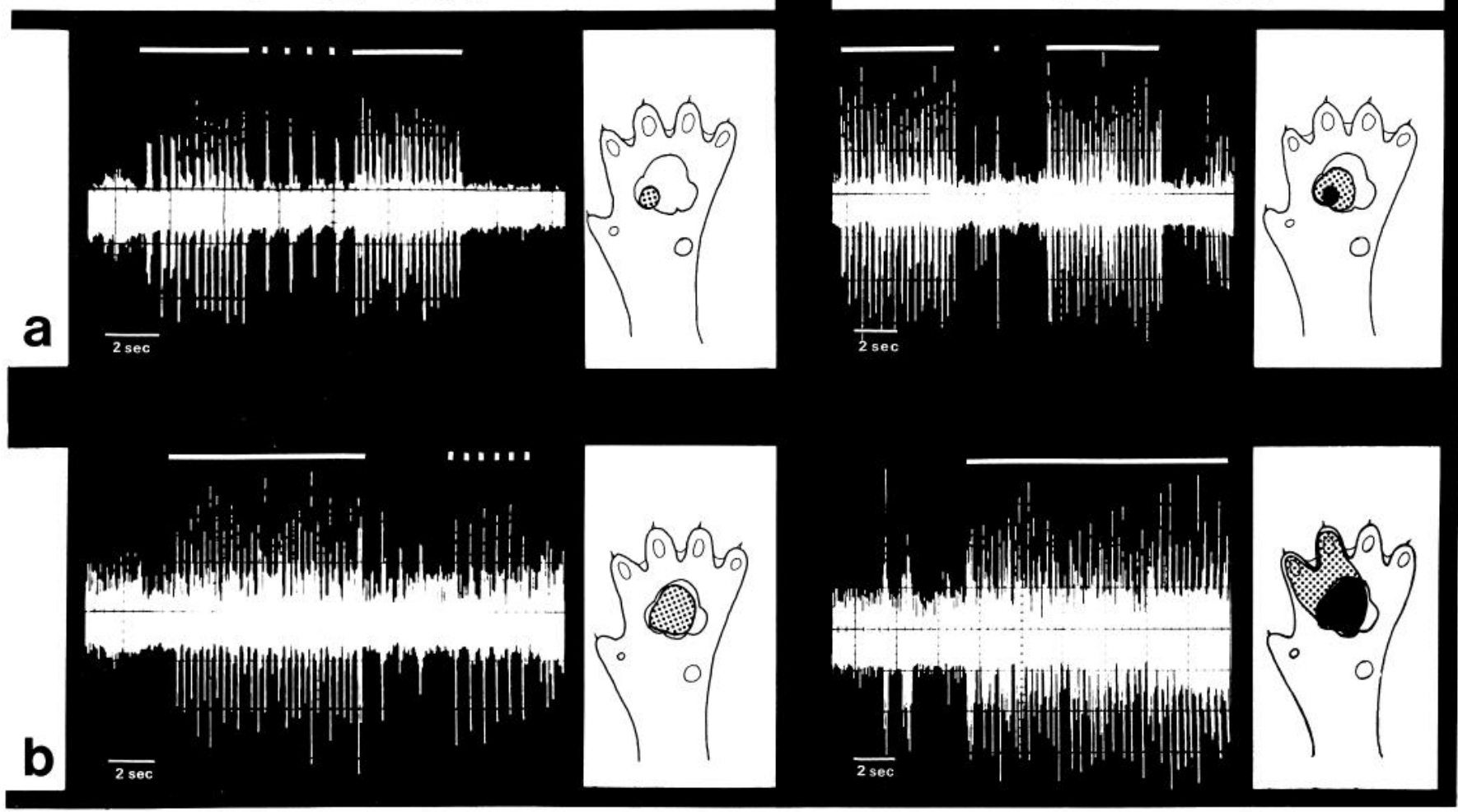

Figure 7. Samples of neural activity obtained from 2 different animals before (pre-BIC) and 30 min after (post-BIC) topical application of BIC to the somatosensory cortex. Panels $a$ and $b$ represent neural activity in 2 separate animals. The white bars above the neural traces indicate the presentation of an intermittent vertical displacement stimulus. White dots indicate the application of a single stimulus. The shaded areas in the paw outlines, in the pre-BIC column indicate the RFs before application of BIC; the shaded areas in the post-BIC column indicate the RFs 30 min after application of $\mathrm{BIC}$, with the original RF in black. See text for details. 


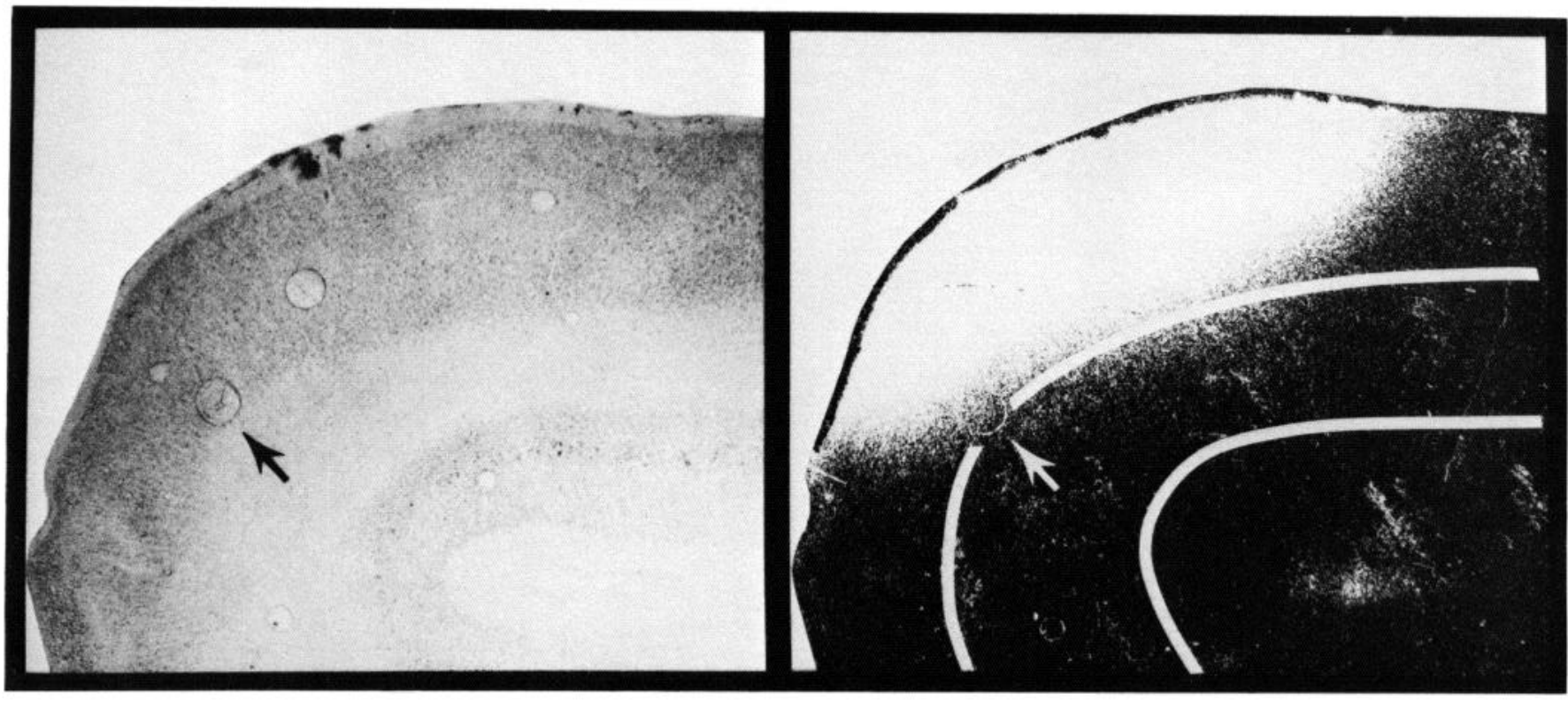

Figure 8. Nissl-stained section (left) and an autoradiograph of the same section (right) illustrating the absorption of ${ }^{3} \mathrm{H}-\mathrm{BIC}$ into the somatosensory cortex. The labeled BIC was applied to the cortex in the same manner as in the 2DG experiments. The image at right was printed directly from the film, and the labeled area is thus white. The inner boundary of the cerebral cortex is indicated with a white line on the autoradiograph. An arrow points to the same artifact (a bubble) on each image. See text for details.

did not reach our criteria for patch definition. The average ${ }^{14} \mathrm{C}$ value for the cortical field surrounding the wide patches was $46 \%$ above background. When lower doses $\left(10^{-6}\right.$ or $\left.10^{-7} \mathrm{M}\right)$ were applied, the abnormally wide patches were again detected, but in this case they were not embedded in a homogeneous field of label (Fig. 11), and were primarily located in the granular and supragranular layers.

The dimensions of the patches observed following application of BIC were measured in the drug-treated hemisphere and compared with the dimensions of patches in the opposite hemisphere. Figure 6 shows that for the hemispheres receiving a $10^{-6}$ M dose of BIC, the stimulus-evoked patches from the 2 stimulation sites averaged 1134 and $1304 \mu \mathrm{m}$ in tangential width, whereas the patches in the opposite, drug-free hemisphere av- eraged 520 and $623 \mu \mathrm{m}$ in width. The SI cortices receiving a $10^{-5} \mathrm{M}$ dose of BIC contained patches averaging 1218 and 1398 $\mu \mathrm{m}$ in width, whereas the opposite hemisphere contained patches averaging 725 and $808 \mu \mathrm{m}$ in tangential width. As a group, the sizes of the patches found after application of BIC were significantly wider (paired $t$ test, $p \leq 0.05$ ) than those in the drugfree opposite hemispheres.

The distributions of metabolic label from both hemispheres of 2 animals that received a $10^{-6} \mathrm{M}$ concentration of BIC on one side were reconstructed as unfolded cortical maps (e.g., Fig. 12). In these animals, the "control" hemispheres (those that did not receive BIC) demonstrated a "typical" pattern of stimulusevoked 2DG activity consisting of intermittent strips. The dimensions of the strips of label were similar to the strips found
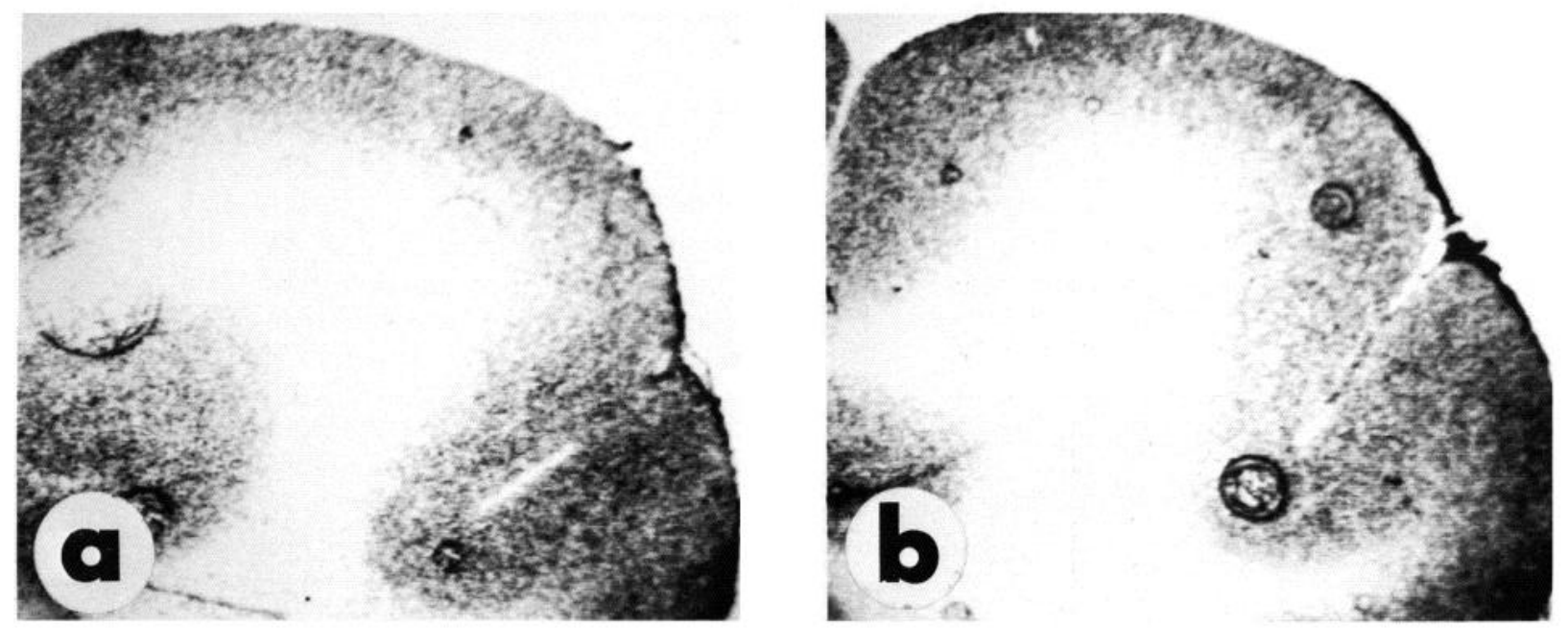

Figure 9. Two digitized autoradiographs taken from rostral $(a)$ and caudal $(b)$ locations in the somatosensory cortex from a brain in which $10^{-6}$ м BIC was applied to the cortex but no somatic stimuli were delivered. No obvious increases in metabolic activity can be observed. Sections in the coronal plane. 

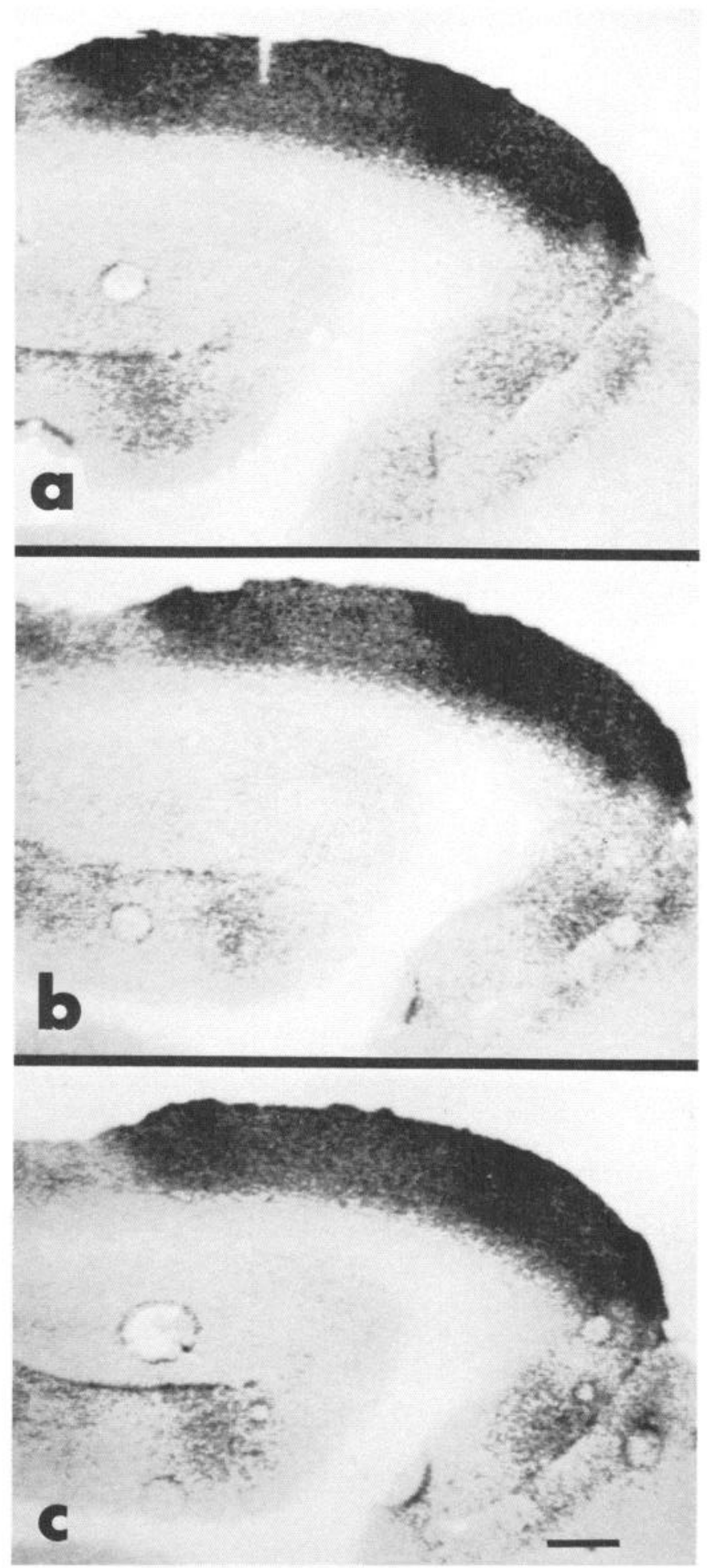

Figure 10. Three digitized autoradiographic sections progressing from rostral $(a)$ to caudal $(c)$ in a brain that received a topical application of $10^{-6} \mathrm{M}$ of BIC and electrocutaneous stimulation to the central and pisiform pads. Abnormally wide patches embedded in a homogeneous field of increased uptake can be observed. Sections in the coronal plane; scale bar, $1 \mathrm{~mm}$.

in animals without pharmacologic manipulation. In addition, the overall distribution of label appeared consistent with published maps of the representation of these body parts.

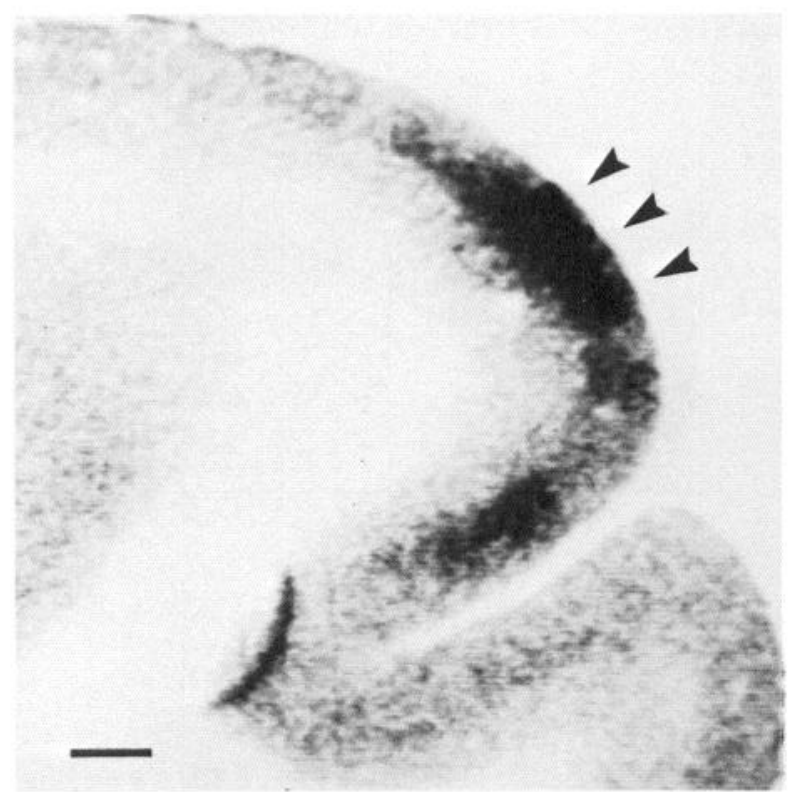

Figure 11. Digitized autoradiograph taken from the somatosensory cortex of an animal that received a $10^{-6} \mathrm{M}$ concentration of BIC. Arrows illustrate an abnormally wide patch of increased 2DG uptake; somatic stimulation was an electrocutaneous stimulus to the central and pisiform pads. Scale bar, $1 \mathrm{~mm}$.

Although several characteristics of the distribution of stimulus-evoked labeling found in the BIC-treated hemispheres were similar to the pattern found on the normal side, a number of features appeared substantially altered. In general, the overall metabolic pattern was consistent with the topographic representation of the body part stimulated, and the labeling was distributed over approximately the same cortical regions occupied by labeling in the normal hemisphere. However, whereas the distribution in the untreated hemisphere was distinctly patchy and formed intermittent strips, the individual strips in the treated hemispheres were wider and clearly less independent. Thus, although periodic fluctuations in the spatial distribution of metabolic labeling remain after treatment with BIC, each "strip" on the treated side tends to blend with its neighbor (Fig. 12A). In addition, there are locations in the BIC-treated hemisphere that lack activity demonstrable on the normal side. There are, for instance, strips of label seen in the intact side that are less evident in the BIC hemisphere.

\section{Discussion}

\section{General distribution}

The distributions of the 2DG label from the few body parts stimulated in these studies appear consistent with published maps of the somatosensory cortex based on RF data (McKenna et al., 1981; Felleman et al., 1983). The metabolic labeling evoked from stimulation of digit 2 and the central pad resides in cortical locations similar to those described by McKenna et al. (1981) and Felleman et al. (1983).

In fact, the metabolic patterns found in this study are more coincident with the topographic distributions predicted from the available neurophysiologic maps than are the distributions of metabolic activity found in monkey SI after the delivery of somatic stimulation. There are several possible reasons for this finding. The results presented here were obtained from lightly 

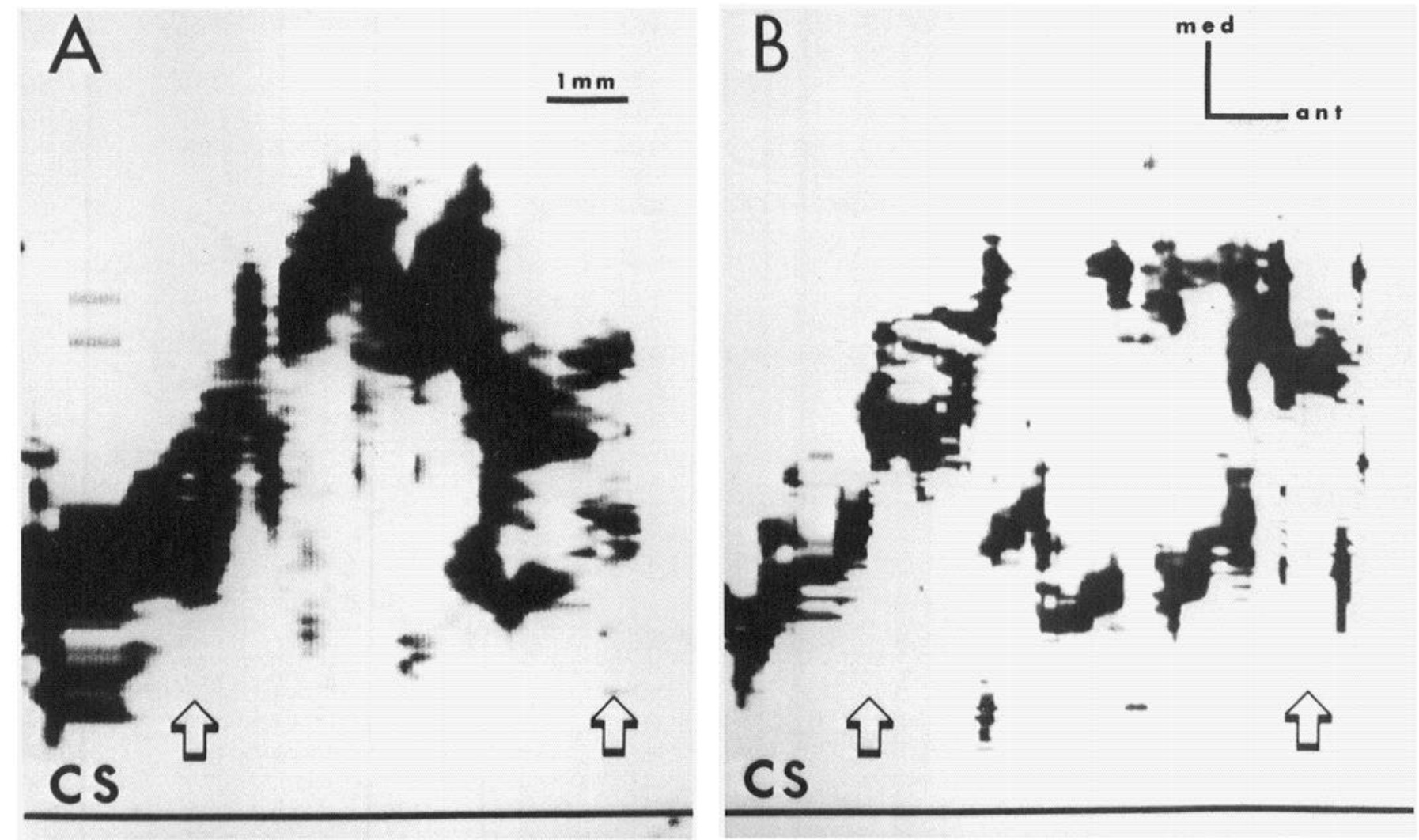

Figure 12. Two digitized reconstructions from both somatosensory cortices of an animal that received a $10^{-6} \mathrm{M}$ topical application of BIC to one hemisphere. $A$, Reconstruction obtained from the side that received BIC. $B$, Control, drug-free hemisphere. The reconstruction shown in $A$ demonstrates strips of labeling that blend with one another and are wider than those in $B$. CS, coronal sulcus. The open arrows indicate equidistant points on each reconstruction. The reconstruction procedure leads to a slightly "blurred" appearance in the unfolded maps.

anesthetized animals, while the activity patterns observed in the monkeys were produced in awake animals. It is well known that anesthetics affect the response properties of neurons in the somatosensory cortex (Collins and Roppolo, 1980; Chapin et al., 1981; McKenna et al., 1981, 1982; Duncan et al., 1982). It may be that in the lightly anesthetized preparation, cortical neuronal activity is suppressed or altered, leading to less glucose consumption and a metabolic map more akin to those obtained with "minimal" RF mapping techniques (Merzenich et al., 1978). Additionally, the stimuli used in these studies were, for the most part, more restricted than those used in the primate studies (Juliano et al., 1981, 1983).

Of interest was the finding that the patches in animals stimulated on the ventral surface of digit 2 were distinctly wider than those obtained from the animals stimulated on the central pad; this was true for both stimulus conditions (i.e., flutter and electrocutaneous). The basis for this difference is not clear. It may be that since the innervation density of the digit 2 pad is greater than that of the central pad, the cortical representation of the ventral surface of digit 2 is larger in size and that the dimensions of $2 \mathrm{DG}$ patches vary with innervation density. Another explanation is that the plane of section used in these studies (coronal) cut across the body map in a manner that was truly coronal or "cross-sectional" for one portion of the map (i.e., central pad) and more oblique for the other distribution (digit 2). Inspection of the map determined by McKenna et al. (1981; see their Fig. 9) indicates that the plane of section could conceivably alter patch width in the manner that it cuts across the body map. Nevertheless, in a study that compares patch dimensions before and after a manipulation (e.g., BIC), it is important that any dependency of patch width on stimulus parameters be recognized.

\section{BIC alters metabolic patches}

When BIC was administered topically to the somatosensory cortex, a pattern of stimulus-evoked 2DG label qualitatively different from that detected in the "normal" subjects was observed. Following BIC, the metabolic patches, normally measuring 500-800 $\mu \mathrm{m}$ in width measured $1.1-1.4 \mathrm{~mm}$. In addition, the regions between the highly activated patches were also often more activated than the interpatch regions found in the "normal" hemispheres. Thus, there appeared to be a "filling in" of the cortical regions activated by the somatic stimulus (i.e., the pattern in this region became less intermittent than the patterns evoked in the absence of BIC). When viewed as an unfolded cortical map, a blurring of the metabolic strips occurred, causing them to be less distinct entities.

The results of this study should be interpreted with caution. Ideally, in demonstrating a pharmacological antagonism one would like to reverse the effect with an agonist. Because of the nature of the $2 \mathrm{DG}$ technique, each experiment allowed only one view of the global response to a prolonged period of somatic stimulation. As a result, we were unable to ascertain if our results using BIC could be antagonized or blocked by GABA or a GABA agonist. Despite these limitations, we believe that the controls carried out allow us to interpret our findings as being the result 
of a localized effect of BIC, because the changes in the patch dimensions were found only during the experiments using BIC and at the locus of the BIC application. In turn, we interpret the effects of BIC on the 2DG label as a consequence of the blockade of GABA (Curtis et al., 1971 a, b; Sillito, 1975a; Dykes et al., 1984).

The control experiments further demonstrate that (1) neural activity is altered in a manner similar to that found in other studies investigating the effects of BIC (Dykes et al., 1984), (2) BIC remains localized to the region to which it is applied and gains access to all cortical layers, and (3) BIC, at the doses used in this study, does not directly act to cause abnormal increases in metabolic labeling but, instead, modifies the pattern of stimulus-evoked activity in the somatosensory cortex.

Recent work involving electrophysiological recording experiments may provide a basis for interpreting the BIC-induced changes observed in the $2 \mathrm{DG}$ labeling. In the visual system, Sillito and colleagues have revealed alterations in the response properties of simple and complex cells in striate cortex after iontophoretic application of BIC (for review, see Sillito, 1984). Simple cells displayed an increase in response magnitude, a loss of discrete "on" and "off" regions in their RFs, and a loss of direction and orientation selectivity (Sillito, 1975a, 1977; Tsumoto et al., 1979). A population of complex cells also demonstrated altered responses and dramatic changes were seen in ocular dominance (Sillito, 1975b, 1977). In general, these results suggest that cells normally responsive to a limited range of stimuli become receptive to a wider field of inputs under the influence of a GABAergic blockade.

Researchers studying the somatosensory cortex of cats have reported that the iontophoretic application of BIC to cells in SI causes an increase in response magnitude to a given stimulus and an expansion of the RF for certain classes of neurons (Hicks and Dykes, 1983; Dykes et al., 1984; Alloway and Burton, 1986). Although changes in modality selectivity were not observed, a number of cells previously not driven by somatic stimulation were "uncovered" to reveal cutaneous receptive fields (Dykes et al., 1984). Studies in cat SII also revealed that neurons in this cortical region expanded their RFs with iontophoretic application of BIC (Alloway and Burton, 1986). Although the neurophysiological observations provided by the experiments of the current study were not extensive, they appear consistent with the reports that BIC increases the RF size of a population of cells in somatosensory cortex. It thus appears that for both the visual and somatosensory cortices, GABA inhibitory transmission focuses the response of individual neurons to a limited range of the numerous inputs that could potentially drive a given cell.

In addition to the general widening and "blurring" of the metabolic pattern after treatment with BIC, we also noted regions of apparently reduced labeling. A portion of the stimulusevoked pattern observed in the normal hemisphere shown in Figure 12 is diminished in the drug-treated pattern. Although (a physiologic) mechanism may exist for explaining these discrepancies, the most likely explanation is individual variation between hemispheres. Even though the distributions of metabolic activity evoked in different hemispheres from apparently identical stimuli are strikingly similar (e.g., Fig. 2, or Juliano and Whitsel, 1985), there are, nevertheless, subtle variations from hemisphere to hemisphere. Indeed, in another experiment in which both hemispheres of a unilaterally BIC-treated animal were reconstructed, such discrepancies between the 2 hemispheres did not occur. Given the complexity of the mammalian cerebral cortex and observations indicating that among normal animals there is a substantial amount of variability in the representation of a given body part (Merzenich et al., 1987), it is not surprising to find slight deviations in the overall pattern between hemispheres. We should also note that although slight stimulus-evoked pattern changes from hemisphere to hemisphere might be recognized, we have never observed dramatic changes in patch width between hemispheres from identical stimuli.

We also noted that the superficial layers of the cortex appeared to be more sensitive to GABAergic blockade than did the lower layers. This was true of both the higher and lower doses of BIC, but at the higher dose $\left(10^{-6} \mathrm{M}\right)$, the abnormally wide patches extended through all layers of the cortex, while with the lower dose $\left(10^{-6} \mathrm{M}\right)$, the width of the patches deviated from normal only in the middle and upper layers. This finding might be explained by the observation that, in primate sensorimotor cortex, neuronal somata that are immunoreactive for GABA are not uniformly distributed throughout the cortical laminae but are more heavily represented in the granular and supragranular layers (Hendry et al., 1987). Additionally, the GABAergic terminations from presumptive inhibitory interneurons in primate sensorimotor cortex are more numerous on pyramidal cells in layer III than on pyramidal cells in layer V (DeFelipe et al., 1985). These data suggest that the upper layers might be more susceptible to GABAergic blockade than the lower layers, leading to stronger labeling in these laminae after BIC application. It is also true that in cat somatosensory cortex the normal patchy pattern of stimulus-evoked 2DG label is primarily located in the upper layers and only occasionally extends into laminae $\mathrm{V}$ and VI. Therefore, the supragranular layers may be intrinsically more susceptible to BIC.

\section{Role of structure in forming metabolic patches}

The 2 stimulus conditions (flutter and electrocutaneous) used in this study were both found to produce 2DG labeling in the form of intermittent patches. Despite general agreement that a columnar organization exists, it has been difficult to define functional somatosensory cortical columns on the basis of RF mapping methods (e.g., Dykes, 1983). A recent study investigating neural responses in the somatosensory cortex of cats suggests that this cortical region is organized in columnar segregates that are definable on the basis of RF (Favorov et al., 1987). Additionally, work investigating the anatomic inputs and outputs to the somatosensory cortex has generated data strongly supportive of an underlying intrinsic patchy or columnar nature. Many of these studies have revealed that the terminal axonal fields and cells of origin for callosal, thalamocortical, and afferent and efferent connections within the somatosensory cortex consist of column-like patches (Kosar and Hand, 1981; for review see Jones, 1983). The functional significance of these structural intermittencies has remained elusive.

Other recent studies have suggested that there may exist an underlying functional organization in somatosensory cortex comprised of alternating bands of multiunit activity that respond in either a rapidly adapting (RA) or a slowly adapting (SA) manner to somatic stimuli (Dykes and Gabor, 1981; Sur et al., 1984). It has been suggested that the anatomic patches in 
the somatosensory cortex represent the termination of a sequence of anatomically segregated parallel pathways carrying RA and SA messages. In fact, in the cat, these functional strips may be closely related to the thalamocortical terminations because they do not actually represent clusters of cells with these properties but, rather, regions of cortex that possess an underlying RA or SA background activity corresponding to regions of thalamic termination (Dykes and Gabor, 1981). In the present experiments, however, the metabolic patches do not appear to depend on RA or SA classes of underlying neural activity because the electrocutaneous stimuli produced well-developed patches despite the fact that such stimuli unselectively activate all classes of large-diameter primary afferent fibers (including RA and SA types).

While a clear physiologic correlate for somatosensory "columns" is lacking, the possibility exists that the structured patterns of efferent and afferent connections to or within the somatosensory cortex may underlie the distribution of metabolic patches. Jones and colleagues have identified structurally and functionally defined units called "rods" in the ventrobasal thalamus (Jones et al., 1982). These workers indicate that the thalamic rods can be defined on the basis of RF and modality properties and that the rods project upon the cortex in a columnlike or strip-like fashion (Friedman and Jones, 1980). Therefore, cortical strips should also be definable on the basis of RF and may provide a structural basis for the metabolic pattern. Whether the anatomically defined strips are actually coincident with the metabolic strips is a subject meriting future investigation. Although patches of afferent, efferent, and intracortical connections clearly exist and presumably have some functional relevance, it is not obvious that a patch of 2DG uptake corresponds to an anatomically defined patch. Experiments addressing this issue involving combination anatomical tracing and 2DG are currently in progress and suggest that intracortical connections are good predictors of the locations of 2DG patches (Juliano et al., 1987).

The finding that, although BIC dramatically changed the patchy 2DG pattern, there always remained an underlying patchlike quality to the labeling supports the idea that metabolic patches arise from focused terminal projections (or cells of origin). In cat somatosensory cortex, the tangential arborization for thalamic afferents has been rcportcd to extend for $1-1.25 \mathrm{~mm}$ (Landry and Deschenes, 1981). This axonal distribution, which is similar to the tangential width measured for the patches produced with BIC, may represent the maximal size for a given "column" in cat SI. During the 2DG experiments in normal somatosensory cortex, this tangential distribution appears not to be fully expressed. The resultant intermittent pattern thus most likely reflects not only the arborization pattern of individual thalamocortical afferents but the further clustering of the terminal arbors into column-like patches (Landry and Deschenes, 1981; Landry et al., 1982), as well as the actions of putative GABAergic inhibitory interneurons. Such neurons have been identified in the somatosensory cortex (Hendry and Jones, 1981; Hendry et al., 1983; Houser et al., 1983) and appear, due to their connections, capable of exerting local and long-range effects. This combination of extrinsic and intrinsic factors may be capable of crcating functional "activity" patterns that are determined by connections. In the case of BIC treatment, we presume that the entire potential cortical representational field (one determined by the distribution of extrinsic excitatory input) is activated, due to the absence of the normal GABAergic shaping that would normally lead to a spatially intermittent pattern.

\section{References}

Alloway, K. D., and H. Burton (1986) Bicuculline-induced alterations in neuronal responses to controlled tactile stimuli in the second somatosensory cortex of the cat: A microiontophoretic study. Somatosensory Res. 3: 197-211.

Chapin, J. K., B. D. Waterhouse, and D. J. Woodward (1981) Differences in cutaneous sensory response properties of single somatosensory cortical neurons in awake and halothane anesthetized rats. Brain Res. Bull. 6: 63-70.

Collins, J. G., and J. R. Roppolo (1980) A comparison of human tactile stimulus velocity discrimination with the ability of SI cortical neurons in awake rhesus monkeys to signal the same velocity differences before and after non-anesthetic doses of pentobarbital. Brain Res. 198: 307-321.

Curtis, D. R., A. W. Duggan, D. Felix, G. A. R. Johnston, and H. McLennan (1971a) Bicuculline, an antagonist of GABA and synaptic inhibition in the spinal cord of the cat. Brain Res. 32: 69-96.

Curtis, D. R., A. W. Duggan, D. Felix, G. A. R. Johnston, and H. McLennan (1971b) Antagonism between bicuculline and GABA in the cat brain. Brain Res. 33: 57-73.

DeFelipe, J., S. H. C. Hendry, E. G. Jones, and D. Schmechel (1985) Variability in the terminations of GABAergic chandelier cell axons on initial segments of pyramidal cell axons in the monkey sensorymotor cortex. J. Comp. Neurol. 231: 364-384.

Duncan, G. H., D. A. Dreyer, T. M. McKenna, and B. L. Whitsel (1982) Dose- and time-dependent effects of ketamine on S-I neurons with cutaneous receptive fields. J. Neurophysiol. 47: 677-699.

Durham, D., and T. A. Woolsey (1978) Acute whisker removal reduces neuronal activity in barrcls of mousc SmI cortex. J. Comp. Neurol. 223: 424-447.

Durham, D., and T. A. Woolsey (1985) Functional organization in cortical barrels of normal and vibrissae-damaged mice: A $\left({ }^{3} \mathrm{H}\right)$ 2-deoxyglucose study. J. Comp. Neurol. 235: 97-110.

Dykes, R. W. (1983) Parallel processing of somatosensory information: A theory. Brain Res. Rev. 6: 47-115.

Dykes, R. W., and A. Gabor (1981) Magnification factors and organization of submodality specific bands in SI cortex of the cat. J. Comp. Neurol. 202: 597-620.

Dykes, R. W., P. Landry, R. Metherate, and T. P. Hicks (1984) Functional role of GABA in cat primary somatosensory cortex: Shaping receptive fields of cortical neurons. J. Neurophysiol. 52: 1066-1093.

Favorov, O. V., M. E. Diamond, and B. L. Whitsel (1987) Evidence for a mosaic representation of the body surface in area $3 \mathrm{~b}$ of the somatic cortex of cat. Proc. Natl. Acad. Sci. USA 84: 6606-6610.

Felleman, D. J., J. T. Wall, C. G. Cusick, and J. Kaas (1983) The representation of the body surface in SI of cats. J. Neurosci. 3: 16481669.

Frieaman, D. P., and E. G. Jones (1980) Focal projection of electrophysiologically defined groupings of thalamic cells on the monkey somatic sensory cortex. Brain Res. 191: 249-252.

Hand, P. J. (1982) Plasticity of the rat cortical barrel system. In Changing Concepts of the Nervous System, P. L. Strick and A. R. Morrison, eds., pp. 49-68, Academic, New York.

Hendry, S. H. C., and E. G. Jones (1981) Sizes and distributions of intrinsic neurons incorporating tritiated GABA in monkey sensorymotor cortex. J. Neurosci. 1: 390-408.

Hendry, S. H. C., C. R. Houser, E. G. Jones, and J. E. Vaughn (1983) Synaptic organization of immunocytochemically identified GABA neurons in the monkey sensory-motor cortex. J. Neurocytol. 12:639660.

Hendry, S. H. C., H. D. Schwark, E. G. Jones, and J. Yan (1987) Numbers and proportions of GABA-immunoreactive neurons in different areas of monkey cerebral cortex. J. Neurosci. 7: 1503-1519.

Hicks, T. P., and R. W. Dykes (1983) Receptive field size for certain neurons in primary somatosensory cortex is determined by GABAmediated intracortical inhibition. Brain Res. 274: 160-164.

Houser, C. R., S. H. C. Hendry, E. G. Jones, and J. E. Vaughn (1983) Morphological diversity of immunocytochemically identified GABA neurons in the monkey sensory-motor cortex. 12: 617-638. 
Jones, E. G. (1983) The columnar basis of cortical circuitry. In The Clinical Neurosciences, Sect. $V$, Neurobiology, R. N. Rosenberg and W. D. Willis, Jr., eds., pp. 357-384, Churchill Livingstone, London.

Jones, E. G., D. P. Friedman, and S. H. C. Hendry (1982) Thalamic basis of place- and modality-specific columns in monkey somatosensory cortex: A correlative anatomical and physiological study. J. Neurophysiol. 48: 545-568.

Juliano, S. L., and B. L. Whitsel (1985) Metabolic labeling assuciated with index finger stimulation in monkey SI: Between animal variability. Brain Res. 342: 242-251.

Juliano, S. L., and B. L. Whitsel (1987) A combined 2-deoxyglucose and neurophysiological study of primate somatosensory cortex. $\mathrm{J}$. Comp. Neurol. 263: 514-525.

Juliano, S. L., P. J. Hand, and B. L. Whitsel (1981) Patterns of increased metabolic activity in somatosensory cortex of monkeys ( $\mathrm{Ma}$ caca fascicularis) subjected to controlled cutaneous stimulation: A 2-deoxyglucose study. J. Neurophysiol. 46: 1260-1284.

Juliano, S. L., P. J. Hand, and B. L. Whitsel (1983) Patterns of metabolic activity in cytoarchitectural area SII and surrounding cortical fields of the monkey. J. Neurophysiol. 50: 961-980.

Juliano, S. L., S. S. Cheema, and B. L. Whitsel (1984) Factors determining patchy metabolic iabeling in the somatosensory cortex of cats. Soc. Neurosci. Abstr. 10: 946.

Juliano, S. L., D. P. Friedman, and D. Eslin (1987) Patterns of corticocortical connectivity can predict patches of stimulus-evoked metabolic activity in monkey somatosensory cortex. Soc. Neurosci. Abstr. 13: 470 .

Kosar, E., and P. J. Hand (1981) First somatosensory cortical columns and associated neuronal clusters of nucleus ventralis posterolateralis of the cat: An anatomical demonstration. J. Comp. Neurol. 198:515539.

Landry, P., and M. Deschenes (1981) Intracortical arborizations and receptive fields of identified ventrobasal thalamocortical afferents to the primary somatic sensory cortex in the cat. J. Comp. Neurol. 199: 345-371.

Landry, P., J. Villemure, and M. Deschenes (1982) Geometry and orientation of thalamocortical arborizations in the cat somatosensory cortex as revealed by computer reconstruction. Brain Res. 237: 222226.

McKenna, T. M., B. L. Whitsel, D. A. Dreyer, and C. B. Metz (1981) Organization of cat anterior parietal cortex: Relations among cytoarchitecture, single neuron functional properties and interhemispheric connectivity. J. Neurophysiol. 45: 667-697.

McKenna, T. M., B. L. Whitsel, and D. A. Dreyer (1982) Anterior parietal cortical topographic organization in macaque monkey: A reevaluation. J. Neurophysiol. 48: 289-317.

Merzenich, M. M., J. H. Kaas, M. Sur, and C. Lin (1978) Double representation of the body surface within cytoarchitectonic areas $3 \mathrm{~b}$ and 1 in "S-I" in the owl monkey (Aotus trivirgatus). J. Comp. Neurol. 181: 41-74.

Merzenich, M. M., R. J. Nelson, J. H. Kaas, M. P. Stryker, W. M. Jenkins, J. M. Zook, M. S. Cynader, and A. Schoppmann (1987) Variability in hand surface representations in areas $3 \mathrm{~b}$ and 1 in adult owl and squirrel monkeys. J. Comp. Neurol. 258: 281-296.

Mountcastle, V. B. (1978) An organizing principle for cerebral function: The unit module and the distributed system. In The Mindful Brain, O. Creutzfeldt, ed., pp. 7-50, MIT Press, Cambridge, MA

Rose, D., and C. Blakemore (1974) Effects of bicuculline on functions of inhibition in visual cortex. Nature 249: 375-377.

Sillito, A. M. (1975a) The effectiveness of bicuculline as an antagonist of GABA and visually evoked inhibition in the cat's striate cortex. J. Physiol. (Lond.) 250: 305-329.

Sillito, A. M. (1975b) The contribution of inhibitory mechanisms to the receptive field properties of neurons in the striate cortex. J. Physiol. (Lond.) 250: 305-329.

Sillito, A. M. (1977) inhibitory processes underlying the directional specificity of simple, complex and hypercomplex cells in the cat's visual cortex. J. Physiol. (Lond.) 271:699-720.

Sillito, A. M. (1984) Functional considerations of the operation of GABAergic inhibitory processes in the visual cortex. In Cerebral Cortex, Vol. 2, Functional Properties of Cortical Cells, E. G. Jones and A. Peters, eds., pp. 91-117, Plenum, New York.

Sokoloff, L., M. Reivich, C. Kennedy, M. H. Des Rosiers, C. S. Patlak, K. D. Pettigrew, O. Sakurada, and M. Shinohara (1977) $\left({ }^{14} \mathrm{C}\right)$ deoxyglucose method for the measurement of local cerebral glucose utilization: Theory, procedure, and normal values in the conscious and anesthetized albino rat. J. Neurochem. 28: 897-916.

Sur, M., J. T. Wall, and J. H. Kaas (1984) Modular distribution of neurons with slowly adapting and rapidly adapting responses in area $3 \mathrm{~b}$ of somatosensory cortex in monkeys. J. Neurophysiol. 51:724744.

Tommerdahl, M., R. Baker, B. L. Whitsel, and S. L. Juliano (1985) A method for reconstructing patterns of somatosensory cerebral cortical activity. Biomed. Sci. Instrum. 21: 93-98.

Tsumoto, T., W. Eckhart, and O. D. Creutzfeldt (1979) Modification of orientation sensitivity of cat visual cortex neurons by removal of GABA-mediated inhibition. Exp. Brain Res. 34: 351-363. 